Lecce University LE/ASTRO-1/97

Pavia University FNT/T-97/4

Zurich University ZU-TH 1/97

\title{
HALO DARK CLUSTERS OF BROWN DWARFS AND MOLECULAR CLOUDS
}

\author{
F. De Paolis ${ }^{1}$, G. Ingrosso ${ }^{2, *}$, Ph. Jetzer $^{1}$ and M. Roncadelli ${ }^{3, \dagger}$ \\ ${ }^{1}$ Paul Scherrer Institute, Laboratory for Astrophysics, CH-5232 Villigen \\ PSI, and Institute of Theoretical Physics, University of Zurich, \\ Winterthurerstrasse 190, CH-8057 Zurich, Switzerland \\ 2 Dipartimento di Fisica, Università di Lecce, Via Arnesano, CP 193, \\ I-73100 Lecce, and INFN, Sezione di Lecce, Via Arnesano, CP 193, I-73100 \\ Lecce, Italy \\ ${ }^{3}$ INFN, Sezione di Pavia, Via Bassi 6, I-27100, Pavia
}

\begin{abstract}
The observations of microlensing events in the Large Magellanic Cloud suggest that a sizable fraction $(\sim 50 \%)$ of the galactic halo is in the form of MACHOs (Massive Astrophysical Compact Halo Objects) with an average mass $\sim 0.27 M_{\odot}$, assuming a standard spherical halo model. We describe a scenario in which dark clusters of MACHOs and cold molecular clouds (mainly of $\mathrm{H}_{2}$ ) naturally form in the halo at galactocentric distances larger than 10-20 kpc.
\end{abstract}

* Partially supported by Agenzia Spaziale Italiana.

$\dagger$ Partially supported by Dipartimento di Fisica Nucleare e Teorica, Università di Pavia, Pavia, Italy.

Talk presented by Ph. Jetzer at the 18th Texas Symposium on Relativistic Astrophysics (Chicago, December 1996). To appear in the proceedings. 


\section{Introduction}

A central problem in astrophysics concerns the nature of the dark matter in galactic halos, whose presence is implied by the flat rotation curves in spiral galaxies. As first proposed by Paczyński [1], gravitational microlensing can provide a decisive answer to that question [2], and since 1993 this dream has started to become a reality with the detection of several microlensing events towards the Large Magellanic Cloud [3, 4. Today, although the evidence for MACHOs is firm, the implications of this discovery crucially depend on the assumed galactic model. It has become customary to take the standard spherical halo model as a baseline for comparison. Within this model, the mass moment method yields an average MACHO mass [5] of $0.27 M_{\odot}$. Unfortunately, because of the presently available limited statistics different data-analysis procedures lead to results which are only marginally consistent. For instance, the average mass reported by the MACHO team is $0.5_{-0.2}^{+0.3} M_{\odot}$. Apart from the low-statistics problem - which will automatically disappear from future larger data samples - we feel that the real question is whether

the standard spherical halo model correctly describes our galaxy [6]. Besides the observational evidence that spiral galaxies generally have flattened halos, recent determinations of the disk scale length, the magnitude and slope of the rotation at the solar position indicate that our galaxy is best described by the maximal disk model. This conclusion is further strengthened by the microlensing results towards the galactic centre, which imply that the bulge is more massive than previously thought. So, the expected average MACHO mass should be smaller than within the standard halo model. Indeed, the value $\sim 0.1 M_{\odot}$ looks as the most realistic estimate to date and suggests that 
MACHOs are brown dwarfs.

\section{Mass moment method}

The most appropriate way to compute the average mass and other important properties of MACHOs is to use the method of mass moments developed by De Rújula et al. [0]. The mass moments $<\mu^{m}>$ are related to $<$ $\tau^{n}>=\sum_{\text {events }} \tau^{n}$, with $\tau \equiv\left(v_{H} / r_{E}\right) T$, as constructed from the observations $\left(v_{H}=210 \mathrm{~km} \mathrm{~s}^{-1}, r_{E}=3.17 \times 10^{9} \mathrm{~km}\right.$ and $T$ is the duration of an event in days). We consider only 6 out of the 8 events observed by the MACHO group during their first two years. In fact, the two disregarded events are a binary lensing and one which is rated as marginal. The ensuing mean mass is $\mathrm{\text {U }}<\mu^{1}>/<\mu^{0}>=0.27 M_{\odot}$, assuming a standard spherical halo model.

Although this value is marginally consistent with the result of the MACHO team, it definitely favours a lower average MACHO mass. For the fraction of the local dark mass density detected in the form of MACHOs, we find $f \sim 0.54$, which compares quite well with the corresponding value $(f \sim 0.45)$ calculated by the MACHO group in a different way.

\section{Formation of dark clusters}

A major problem concerns the formation of MACHOs, as well as the nature of the remaining amount of dark matter in the galactic halo. We feel it hard to conceive a formation mechanism which transforms with $100 \%$ efficiency hydrogen and helium gas into MACHOs. Therefore, we expect that also

\footnotetext{
${ }^{1}$ When taking for the duration $T$ the values corrected for "blending", we get as average mass $0.34 M_{\odot}$. We thank D. Bennett for driving our attention on this point.
} 
cold clouds (mainly of $\mathrm{H}_{2}$ ) should be present in the galactic halo. Recently, we have proposed a scenario [8] in which dark clusters of MACHOs and cold molecular coulds naturally form in the halo at galactocentric distances larger than 10-20 kpc, with the relative abundance possibly depending on the distance.

The evolution of the primordial proto globular cluster clouds (which make up the proto-galaxy) is expected to be very different in the inner and outer parts of the Galaxy, depending on the decreasing ultraviolet flux (UV) from the centre as the galactocentric distance $R$ increases. In fact, in the outer halo no substantial $H_{2}$ depletion should take place, owing to the distance suppression of the UV flux. Therefore, the clouds cool and fragment - the process stops when the fragment mass becomes $\sim 10^{-2}-10^{-1} M_{\odot}$. In this way dark clusters should form, which contain brown dwarfs and also cold $\mathrm{H}_{2}$ self-gravitating cloud, along with some residual diffuse gas (the amount of diffuse gas inside a dark cluster has to be low, for otherwise it would have been observed in the radio band).

We have also considered several observational tests for our model [8, 9]. In particular, a signature for the presence of molecular clouds in the galactic halo should be a $\gamma$-ray flux produced in the scattering of high-energy cosmic-ray protons on $H_{2}$. As a matter of fact, an essential information is the knowledge of the cosmic ray flux in the halo. Unfortunately, this quantity is unknown and the only available information comes from theoretical considerations. Nevertheless, we can make an estimate of the expected $\gamma$-ray flux and the best chance to detect it is provided by observations at high galactic latitude. Accordingly, we find a $\gamma$-ray flux (for $E_{\gamma}>100 \mathrm{MeV}$ ) 
$\Phi_{\gamma}\left(90^{0}\right) \simeq 1.1 \times 10^{-6} \epsilon f$ photons $\mathrm{cm}^{-2} \mathrm{~s}^{-1} \mathrm{sr}^{-1}(\epsilon$ is a unknown parameter which takes into account the degree of confinement of the cosmic rays in the halo, whereas $f$ stands for the fraction of halo dark matter in the form of cold molecular gas). This flux should be compared with the measured value for the diffuse background of $0.7-2.3 \times 10^{-5}$ photons $\mathrm{cm}^{-2} \mathrm{~s}^{-1} \mathrm{sr}^{-1}$. Thus, there is at present no contradiction with observations. Furthermore, an improvement of sensitivity for the next generation of $\gamma$-ray detectors will either discover the effect in question or yield more stringent limits on $\epsilon f$.

\section{References}

[1] B. Paczyński, Astrophys. J. 304, 1 (1986).

[2] A. De Rújula, Ph. Jetzer and E. Massó, Astron. Astrophys. 254, 99 (1992).

[3] C. Alcock et al., Nature 365, 621 (1993); astro-ph 9606165.

[4] E. Aubourg et al., Nature 365, 623 (1993).

[5] Ph. Jetzer, Helv. Phys. Acta 69, 179 (1996).

[6] F. De Paolis, G. Ingrosso and Ph. Jetzer, Astrophys. J. 470, 493 (1996).

[7] A. De Rújula, Ph. Jetzer and E. Massó, Mont. Not. R. Astr. Soc. 250, 348 (1991).

[8] F. De Paolis, G. Ingrosso, Ph. Jetzer and M. Roncadelli, Phys. Rev Lett. 74, 14 (1995); Astron. Astrophys. 295, 567 (1995); Comments 
on Astrophys. 18, 87 (1995); Astrophys. and Space Science 235, 329 (1996); Int. J. Mod. Phys. D5, 151 (1996).

[9] F. De Paolis, G. Ingrosso, Ph. Jetzer, A. Qadir and M. Roncadelli, Astron. Astrophys. 299, 647 (1995). 